\title{
Ultrasonic Attenuation in Calcium Oxide
}

\author{
Jitendra Kumar ${ }^{1}$, Kailash $^{2}$, Sanjeev Kumar Shrivastava ${ }^{3}$, Devraj Singh ${ }^{4}$, Virendra Kumar ${ }^{2}$ \\ ${ }^{1}$ Department of Physics, Govt. Girls P. G. College, Banda, India \\ ${ }^{2}$ Department of Physics, BN PG College, Rath, Hamirpur, India \\ ${ }^{3}$ Department of Physics, Bundelkhand University, Jhansi, India \\ ${ }^{4}$ Department of Applied Physics, AMITY School of Engineering and Technology, New Delhi, India \\ E-mail: \{jkumarsh, kailashrath\}@gmail.com \\ Received July 4, 2011; revised August 15, 2011; accepted August 26, 2011
}

\begin{abstract}
Ultrasonic attenuation studies can be used to characterize material not only after production but during processing as well. The most important causes of ultrasonic attenuation in solids are electron-phonon, phonon-phonon interaction and that due to thermo elastic relaxation. The two dominant processes that will give rise to appreciable ultrasonic attenuation at higher temperature are the phonon-phonon interaction also known as Akhiezer loss and that due to thermo elastic relaxation are observed in calcium oxide crystal. At frequencies of ultrasonic range and at higher temperatures in solids, phonon-phonon interaction mechanism is dominating cause for attenuation. Ultrasonic attenuation due to phonon-phonon interaction $\left(\alpha / \mathrm{f}^{2}\right)_{\mathrm{p}-\mathrm{p}}$ and thermo elastic relaxation $\left(\alpha / \mathrm{f}^{2}\right)_{\text {th }}$ are evaluated in Calcium Oxide crystal up to an elevated temperature from $100 \mathrm{~K}-1500 \mathrm{~K}$ along $<100>,<110>$ and $<111>$ crystallographic directions. Temperature dependence of ultrasonic attenuation along different crystallographic direction reveals some typical characteristic features.
\end{abstract}

Keywords: Attenuation, Thermo Elastic Relaxation, Phonon-Phonon Interaction, Akheiser Loss, Gruneisen Constants

\section{Introduction}

Ultrasonic velocity and attenuation parameters are well connected to the micro structural and mechanical properties of the materials. In recent years, the ultrasonic attenuation techniques [1-4] are widely used as versatile tool in studying the inherent properties and internal structure of solids. In several types of solids, viz. metallic dielectric and semiconducting crystals, the attenuation occurs due to various causes e.g. lattice imperfection, ferromagnetic and ferroelectric, NMR and thermal relaxation and thermoelastic loss at different temperature regions. The temperature dependent part of ultrasonic attenuation has been explained in terms of model where the acoustic phonon interacts with a number of thermal phonons in the lattice. The most important causes of ultrasonic attenuation in solids are electron-phonon, phonon-phonon interaction and that due to thermo elastic relaxation. The ultrasonic attenuation study of the materials has gained new dimensions with the progress in the material science. In recent years, the ultrasonic attenuation techniques [5-7] are widely used as versatile tool in studying the inherent properties and internal structure of solids. The two dominant processes that will give rise to appreciable ultrasonic attenuation at higher temperature are the phonon-phonon interaction also known as Akhieser loss $[8,9]$ and that due to thermo elastic relaxation and are observed in calcium oxide crystal.

Oxides and silicates make up the bulk of the Earth's mantle and crust, and thus it is useful to predict their behaviour. In this work ultrasonic attenuation due to phonon-phonon interaction over frequency $\left(\alpha / \mathrm{f}^{2}\right)_{\mathrm{Akh}}$ and ultrasonic attenuation due to thermo elastic relaxation over frequency $\left(\alpha / \mathrm{f}^{2}\right)_{\text {th }}$ are studied in calcium oxide at an elevated temperatures $(100 \mathrm{~K}-1500 \mathrm{~K})$ along $<100>$, $<110>$ and $<111>$ crystallographic directions. For the evaluation of the ultrasonic coefficients the second and third order elastic constants (SOECs and TOECs) are also calculated using Coulomb and Born Mayer [10] potentials. Several investigators have given different theories; here the one given by Mason has been used. Mason's theory relates the Gruneisen constants with SOECs and TOECs. The behaviour of ultrasonic absorption and other parameters as a function of higher temperature have been discussed as the characteristic features of calcium oxide. 


\section{Formulation}

The calcium oxide possesses face centered cubic crystal structure. The potential used for evaluation of second and third order elastic constants (SOECs and TOECs) is taken as the sum of Coulomb and Börn-Mayer potentials.

$$
\mathrm{Q}(\mathrm{r})=\mathrm{Q}(\mathrm{C})+\mathrm{Q}(\mathrm{B})
$$

where $\mathrm{Q}(\mathrm{C})$ is the Coulomb potential and $\mathrm{Q}(\mathrm{B})$ is the Bö rn-Mayer potential, given as

$$
\mathrm{Q}(\mathrm{C})=\{ \pm(\mathrm{e} 2 / \mathrm{r})\} \text { and } \mathrm{Q}(\mathrm{B})=\mathrm{A} \exp (-\mathrm{r} / \mathrm{q})
$$

where e is the electronic charge, $r$ is the nearest-neighbour distance, $\mathrm{q}$ is the hardness parameter and $\mathrm{A}$ is the strength parameter.

Following Brügger's [11] definition of elastic constants at absolute zero, second and third order elastic constants (SOECs and TOECs) are obtained. According to lattice dynamics developed by Leibfried and Ludwig, lattice energy changes with temperatures. Hence adding vibrational energy contribution to the static elastic constants, one gets second and third order elastic constants $\left(\mathrm{C}_{\mathrm{ij}}\right.$ and $\left.\mathrm{C}_{\mathrm{ijk}}\right)$ at the required temperature.

$$
\mathrm{C}_{\mathrm{ij}}=\mathrm{C}_{i \mathrm{j}}^{0}+\mathrm{C}_{\mathrm{ij}}^{\mathrm{vib}} \text { and } \mathrm{C}_{\mathrm{ijk}}=\mathrm{C}_{i \mathrm{jk}}^{0}+\mathrm{C}_{\mathrm{ijk}}^{\mathrm{vib}}
$$

The expressions for ultrasonic attenuation coefficient due to phonon-phonon interaction [12] over frequency square are as

for longitudinal waves

$$
\left(\alpha / \mathrm{f}^{2}\right)_{\mathrm{Akh}}=\frac{4 \pi^{2} \mathrm{E}_{0}\left(\frac{\mathrm{D}_{1}}{3}\right) \tau_{1}}{2 \rho \mathrm{V}_{1}^{3}}
$$

for shear waves

$$
\left(\alpha / \mathrm{f}^{2}\right)_{\mathrm{Akh}}=\frac{4 \pi^{2} \mathrm{E}_{0}\left(\frac{\mathrm{D}_{\mathrm{s}}}{3}\right) \tau_{\mathrm{s}}}{2 \rho \mathrm{V}_{\mathrm{s}}^{3}}
$$

where the condition $\omega \tau_{\text {th }}<<1$ has already been assumed. Here $E_{0}$ is thermal energy density, $\omega$ is the angular frequency $(=2 \pi \mathrm{f})$, $\mathrm{f}$ is frequency of ultrasonic waves, $\mathrm{d}$ is the density and $V_{l}, V_{s}$ are the ultrasonic velocities for longitudinal and shear waves respectively, and the ultrasonic attenuation coefficient due to thermal relaxation over frequency

$$
\left(\alpha / \mathrm{f}^{2}\right)_{\text {th }}=\frac{4 \pi^{2}\left\langle\gamma_{\mathrm{i}}^{\mathrm{j}}\right\rangle^{2} \mathrm{KT}}{2 \rho \mathrm{V}^{5}}
$$

where the condition $\omega \tau_{\text {th }}<<1$ has already been assumed. Here $E_{0}$ is thermal energy density, $f$ is frequency of ultrasonic waves, $\mathrm{d}$ is the density and $\mathrm{V}_{\mathrm{l}}, \mathrm{V}_{\mathrm{s}}$ are the ultrasonic velocities for longitudinal and shear waves respectively.

Two relaxation times are related as

$$
\frac{1}{2} \tau_{1}=\tau_{\mathrm{s}}=\tau_{\mathrm{th}}=\frac{3 \mathrm{~K}}{\mathrm{C}_{\mathrm{V}} \overline{\mathrm{V}}_{\mathrm{D}}^{2}}
$$

where $\tau_{\text {th }}$ is the thermal relaxation time for the exchange of acoustic and thermal energy, $\mathrm{K}$ is the thermal conductivity, $\mathrm{C}_{\mathrm{v}}$ is specific heat and $\overline{\mathrm{V}_{\mathrm{D}}}$ is the Debye average velocity.

$\mathrm{D}$ in eqs. (1) and (2) is given as

$$
\mathrm{D}=9\left\langle\left(\gamma_{\mathrm{i}}^{\mathrm{j}}\right)^{2}\right\rangle-\left(\frac{3 \mathrm{C}_{\mathrm{v}} \mathrm{T}}{\mathrm{E}_{0}}\right)\left\langle\gamma_{\mathrm{i}}^{\mathrm{j}}\right\rangle^{2}
$$

where $\left\langle\left(\gamma_{i}^{\mathrm{j}}\right)^{2}\right\rangle$ and $\left\langle\gamma_{\mathrm{i}}^{\mathrm{j}}\right\rangle^{2}$ are the square average Grüneisen parameters and average square Grüneisen parameters respectively.

\section{Evaluation}

The SOECs $\left(\mathrm{C}_{\mathrm{ij}}\right)$ and TOECs $\left(\mathrm{C}_{\mathrm{ijk}}\right)$ for Calcium oxide has been evaluated at different temperatures $(100 \mathrm{~K}$ to 1500 $\mathrm{K})$ following Brugger's approach and are presented in Table 1 at room temperature. Grüneisen parameters have been evaluated using Mason's Grüneisen parameters tables [13] at different temperatures along different crystallographic directions longitudinal (long.) as well as shear with the help of SOECs and TOECs. The nonlinearity constants (D) are evaluated using the average square Grüneisen parameters $\left\langle\gamma_{i}^{j}\right\rangle^{2}$ and square average Grüneisen parameters $\left\langle\left(\gamma_{\mathrm{i}}^{\mathrm{j}}\right)^{2}\right\rangle$. The values of $\left\langle\left(\gamma_{\mathrm{i}}^{\mathrm{j}}\right)^{2}\right\rangle$, $\left(\alpha / \mathrm{f}^{2}\right)_{\mathrm{Akh}}$ and $\left(\alpha / \mathrm{f}^{2}\right)_{\text {th }}$ along with $\mathrm{V}$ for longitudinal and shear waves, $\tau_{\text {th }}$ and $\mathrm{D}$ are calculated using primary physical data presented in Table 2 and are given in Table 3 at room temperature along different crystallographic directions. The temperature variations of all these properties are presented graphically in Figures 1-6. All these calculations have been done using $\mathrm{C}++$ programmes developed by

Table 1. The second and third order elastic constants in $10^{10} \mathrm{~N} / \mathrm{m}^{2}$ at room temperature alongwith melting point.

\begin{tabular}{cccccccccc}
\hline Melting Point (K) & $\mathrm{C}_{11}$ & $\mathrm{C}_{12}$ & $\mathrm{C}_{44}$ & $\mathrm{C}_{111}$ & $\mathrm{C}_{112}$ & $\mathrm{C}_{123}$ & $\mathrm{C}_{144}$ & $\mathrm{C}_{166}$ & $\mathrm{C}_{456}$ \\
\hline 2843 & 20.67 & 12.52 & 12.71 & -252.88 & -65.10 & 20.34 & 19.43 & -51.26 & 4.71 \\
\hline
\end{tabular}


Table 2. Primary Physical Data (Calculated).

\begin{tabular}{cccc}
\hline Temperature $(\mathrm{K})$ & $\theta_{\mathrm{D}} / \mathrm{T}$ & $\mathrm{C}_{\mathrm{v}}\left(\right.$ in $\left.10^{6} \mathrm{~J} / \mathrm{m}^{3} . \mathrm{K}\right) \mathrm{E}_{0}\left(\right.$ in $\left.10^{6} \mathrm{~J} / \mathrm{m}^{3}\right)$ \\
\hline 100 & 6.480 & 0.3365991 & 9.524686 \\
200 & 3.240 & 0.9255680 & 75.724882 \\
300 & 2.160 & 1.1894222 & 183.37022 \\
400 & 1.620 & 1.3075118 & 308.93472 \\
500 & 1.296 & 1.3540948 & 444.12383 \\
600 & 1.080 & 1.4023710 & 581.71147 \\
700 & 0.925 & 1.4238437 & 723.34339 \\
800 & 0.810 & 1.4379470 & 866.22996 \\
900 & 0.720 & 1.4477110 & 1010.6013 \\
1000 & 0.648 & 1.4547245 & 1155.8323 \\
1100 & 0.589 & 1.4601010 & 1301.5421 \\
1200 & 0.540 & 1.4640190 & 1447.8532 \\
1300 & 0.498 & 1.4673480 & 1594.5340 \\
1400 & 0.462 & 1.4697214 & 1741.9166 \\
1500 & 0.432 & 1.4716960 & 1888.4134 \\
\hline
\end{tabular}

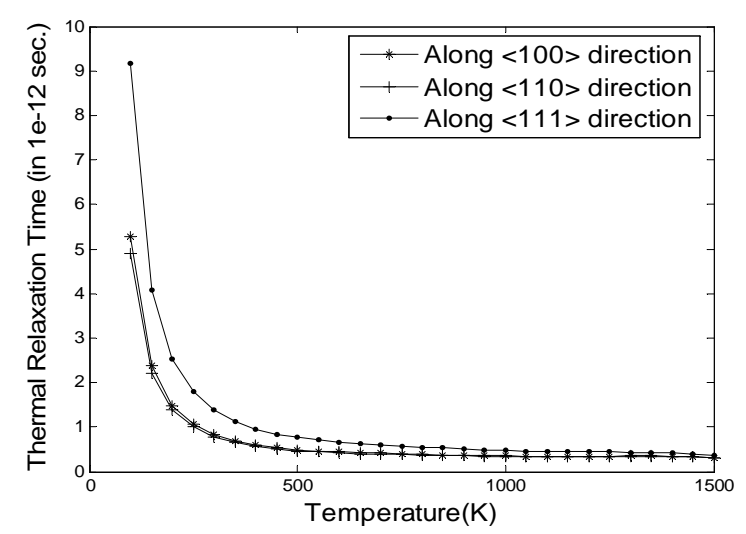

Figure 1. Thermal relaxation time Vs Temp.

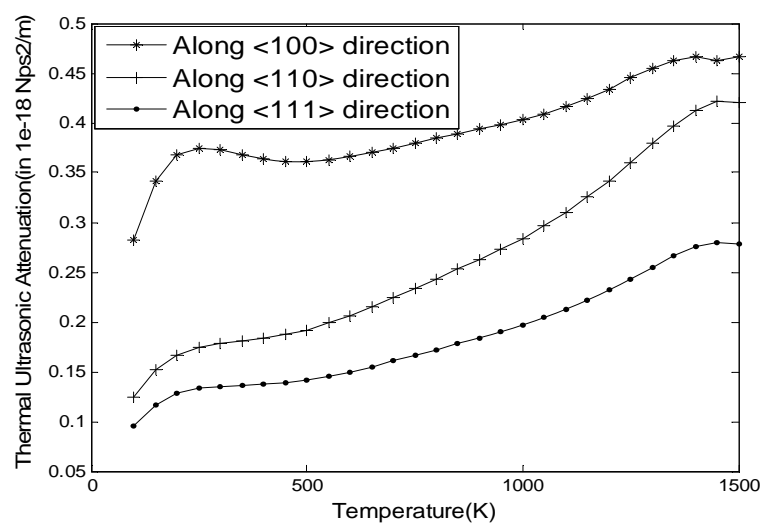

Figure 3. Thermal ultrasonic attenuation Vs Temp.
Table 3. Long. and shear wave velocities in $10^{3} \mathrm{~m} / \mathrm{sec}$ thermal relaxation time in $10^{-12} \mathrm{sec}$, non-linearity constants, thermal attenuation in $10^{-18} \mathrm{~Np} . \mathrm{sec}^{2} / \mathrm{m}$ and $\mathrm{p}$-p attenuation for long and shear waves in $10^{-16} \mathrm{~Np} . \mathrm{sec}^{2} / \mathrm{m}$ at room temperature.

\begin{tabular}{|c|c|c|c|c|c|c|}
\hline $\begin{array}{l}\text { Direction of } \\
\text { propagation }\end{array}$ & $\left\langle\left(\gamma_{i}^{j}\right)^{2}\right\rangle$ & V & $\tau_{\mathrm{th}}$ & $\mathrm{D}$ & $\left(\alpha / \mathrm{f}^{2}\right)_{\mathrm{th}}$ & $\left(\alpha / \mathrm{f}^{2}\right)_{\mathrm{Akh}}$ \\
\hline $\begin{array}{l}<100> \\
\text { Long. }\end{array}$ & 1.947 & 7.520 & 0.836 & 15.414 & 0.373 & 0.218 \\
\hline Shear & 0.321 & 6.171 & - & 2.886 & - & 0.037 \\
\hline $\begin{array}{c}<110> \\
\text { Longitudinal }\end{array}$ & 1.655 & 9.124 & 0.782 & 12.248 & 0.178 & 0.091 \\
\hline $\begin{array}{c}\text { Shear } \\
(\text { pol.<001>) }\end{array}$ & 0.642 & 6.171 & - & 5.777 & - & 0.069 \\
\hline$($ pol. $<1 \overline{1} 0>)$ & 1.538 & 3.375 & - & 13.838 & - & 6.253 \\
\hline $\begin{array}{c}<111> \\
\text { Longitudinal }\end{array}$ & 1.855 & 9.599 & 1.379 & 14.115 & 0.135 & 0.158 \\
\hline $\begin{array}{c}\text { Shear } \\
(\text { pol. }<\overline{1} 10>)\end{array}$ & 0.976 & 4.504 & - & 8.786 & - & 0.954 \\
\hline$($ pol. $<11 \overline{2}>)$ & 1.503 & 4.504 & - & 13.529 & - & 1.469 \\
\hline
\end{tabular}

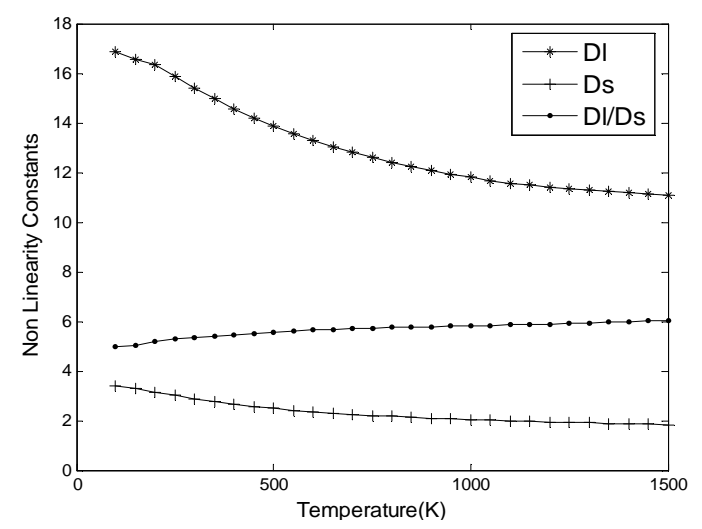

Figure 2. Non-linearity constants Vs Temp. along $<100>$.

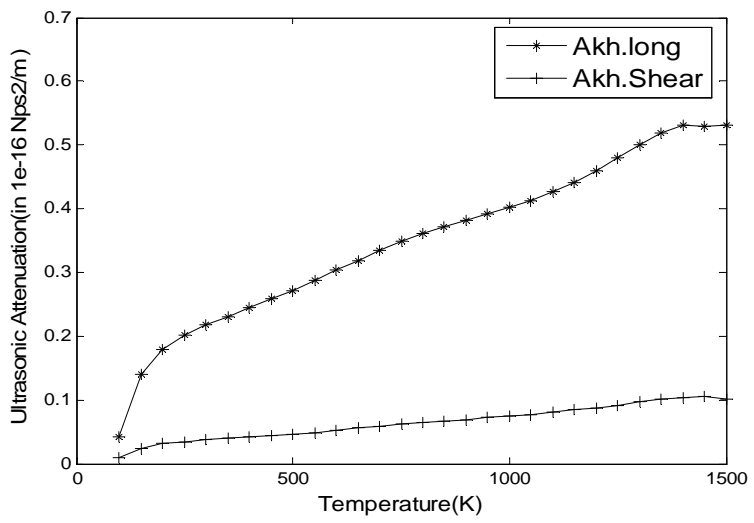

Figure 4. Ultrasonic attenuation Vs Temp. along $<100>$. 
author itself. The developed programmes have been verified using the known results for other FCC structured solids. The Debye temperature $\left(\theta_{\mathrm{D}}\right)$ is an important physical parameter of solids, which defines a division line between quantum and classical behaviour of phonons, its value for $\mathrm{CaO}$ crystal is $648 \mathrm{~K}$. Interpretation of data for ultrasonic attenuation shown in figures is presented in Table 4 through curve fitting.

\section{Results and Discussion}

Table 1 shows that all the SOECs are positive in nature. Among TOECs $\mathrm{C}_{111}, \mathrm{C}_{11} 2$ and $\mathrm{C}_{166}$ are positive and all others are negative in nature. These results are in good agreement with the results obtained for other rocksalt structure solids. The shear waves have a relaxation time

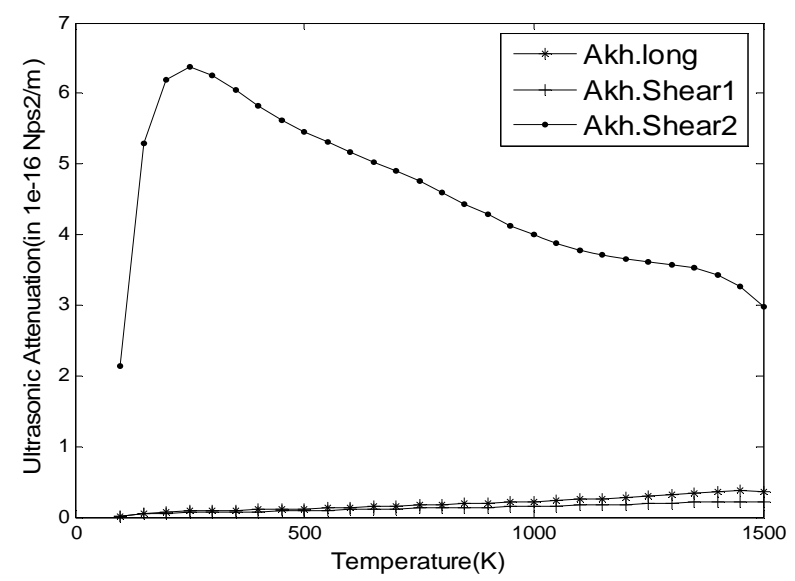

Figure 5. Ultrasonic attenuation Vs Temp. along $<110>$. equal to the thermal relaxation time, while longitudinal waves have a relaxation time about twice as large. Hence, in determining the ultrasonic attenuation due to conversion to thermal phonons, it is necessary to weight the conversion coefficients by this difference in the relaxation time. It is clear from the Figure $\mathbf{1}$ that the value of thermal relaxation time $\tau_{\text {th }}$ is very high at low temperature and decreases as temperature is increased along all crystallographic directions and of the order of $10^{-12} \mathrm{sec}$, which is also expected. Its value is very much at $100 \mathrm{~K}$ but as temperature increases it decreases very sharply up to 500 $\mathrm{K}$ and after that it decreases very slowly up to $1500 \mathrm{~K}$. At any particular temperature its value is more along $<111>$ direction. From the values of thermal relaxation time, we can check the validity of the condition $\omega \tau_{\text {th }}<<1$ in the ultrasonic frequency range.

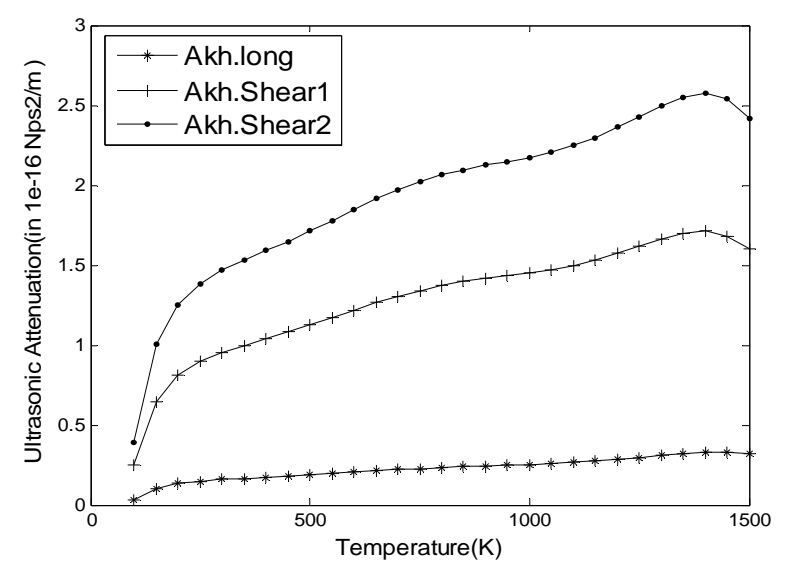

Figure 6. Ultrasonic attenuation Vs Temp. along $<111>$.

Table 4. Property $(P)=A_{0}+A_{1} T+A_{2} T^{2}+A_{3} T^{3}+A_{4} T^{4}+A_{5} T^{5}+A_{6} T^{6}+A_{7} T^{7}+A_{8} T^{8}+A_{9} T^{9}+A_{10} T^{10}$ for Ultrasonic Attenuation.

\begin{tabular}{|c|c|c|c|c|c|c|c|c|c|c|c|}
\hline $\mathrm{P}$ & $\mathrm{A}_{0}$ & $\mathrm{~A}_{1}$ & $\mathrm{~A}_{2}$ & $\mathrm{~A}_{3}$ & $\mathrm{~A}_{4}$ & $\mathrm{~A}_{5}$ & $\mathrm{~A}_{6}$ & $\mathrm{~A}_{7}$ & $\mathrm{~A}_{8}$ & $\mathrm{~A}_{9}$ & $\mathrm{~A}_{10}$ \\
\hline$\left(\alpha / \mathrm{f}^{2}\right)_{\mathrm{th}<100>}$ & $-1.52 \mathrm{e}-02$ & $4.59 \mathrm{e}-02$ & $-1.90 \mathrm{e}-05$ & $2.60 \mathrm{e}-08$ & $4.23 \mathrm{e}-11$ & $-2.21 \mathrm{e}-13$ & $3.93 e-16$ & $-3.89 \mathrm{e}-19$ & $2.27 \mathrm{e}-22$ & $-7.30 \mathrm{e}-26$ & $9.99 \mathrm{e}-30$ \\
\hline$\left(\alpha / \mathrm{f}^{2}\right)_{\mathrm{Akh} .1<100>}$ & -0.59 & $1.17 \mathrm{e}-02$ & $-7.76 \mathrm{e}-05$ & $2.97 \mathrm{e}-07$ & $-7.13 e-10$ & $1.12 \mathrm{e}-12$ & $-1.16 \mathrm{e}-15$ & $7.85 \mathrm{e}-19$ & $-3.28 \mathrm{e}-22$ & $7.64 \mathrm{e}-26$ & $-7.48 \mathrm{e}-30$ \\
\hline$\left(\alpha / \mathrm{f}^{2}\right)_{\text {Akh.s }<100>}$ & $-9.27 \mathrm{e}-02$ & $1.91 \mathrm{e}-02$ & $-1.31 \mathrm{e}-05$ & $5.33 \mathrm{e}-08$ & $-1.38 \mathrm{e}-10$ & $2.38 \mathrm{e}-13$ & $-2.75 e-16$ & $2.10 \mathrm{e}-19$ & $-1.01 \mathrm{e}-22$ & $2.80 \mathrm{e}-26$ & $-3.36 \mathrm{e}-30$ \\
\hline$\left(\alpha / \mathrm{f}^{2}\right)_{\mathrm{th}<110>}$ & $-7.10 \mathrm{e}-02$ & $2.06 \mathrm{e}-02$ & $-9.64 \mathrm{e}-06$ & $2.47 \mathrm{e}-08$ & $-3.93 e-11$ & $4.42 \mathrm{e}-14$ & $-3.75 e-17$ & $2.35 \mathrm{e}-20$ & $-9.98 \mathrm{e}-24$ & $2.48 \mathrm{e}-27$ & $-2.79 e-31$ \\
\hline$\left(\alpha / \mathrm{f}^{2}\right)_{\mathrm{Akh} .1<110>}$ & -0.25 & $4.97 e-03$ & $-3.37 \mathrm{e}-05$ & $1.34 \mathrm{e}-07$ & $-3.41 \mathrm{e}-10$ & $5.75 e-13$ & $-6.48 \mathrm{e}-16$ & $4.81 \mathrm{e}-19$ & $-2.25 \mathrm{e}-22$ & $6.04 \mathrm{e}-26$ & $-7.04 \mathrm{e}-30$ \\
\hline$\left(\alpha / \mathrm{f}^{2}\right)_{\mathrm{Akh} . \mathrm{s} 1<110>}$ & -0.14 & $3.03 \mathrm{e}-03$ & $-1.99 \mathrm{e}-05$ & $7.78 \mathrm{e}-08$ & $-1.91 \mathrm{e}-10$ & $3.10 \mathrm{e}-13$ & $-3.32 \mathrm{e}-16$ & $2.31 \mathrm{e}-19$ & $-9.90 \mathrm{e}-23$ & $2.34 \mathrm{e}-26$ & $-2.31 \mathrm{e}-30$ \\
\hline$\left(\alpha / \mathrm{f}^{2}\right)_{\text {Akh.s } 2<110>}$ & -22.14 & 0.46 & $-3.25 \mathrm{e}-03$ & $1.27 \mathrm{e}-05$ & $-3.18 \mathrm{e}-08$ & $5.26 \mathrm{e}-11$ & $-5.82 \mathrm{e}-14$ & $4.26 \mathrm{e}-17$ & $-1.98 \mathrm{e}-20$ & $5.29 \mathrm{e}-24$ & $-6.16 e-28$ \\
\hline$\left(\alpha / \mathrm{f}^{2}\right)_{\mathrm{th}<111>}$ & $-1.16 \mathrm{e}-02$ & $1.69 \mathrm{e}-03$ & $-8.06 \mathrm{e}-06$ & $2.05 \mathrm{e}-08$ & $-3.16 \mathrm{e}-11$ & $3.25 \mathrm{e}-14$ & $-2.42 \mathrm{e}-17$ & $1.35 \mathrm{e}-20$ & $-5.36 \mathrm{e}-24$ & $1.32 \mathrm{e}-27$ & $-1.56 \mathrm{e}-31$ \\
\hline$\left(\alpha / \mathrm{f}^{2}\right)_{\mathrm{Akh} .1<111>}$ & -0.51 & $1.04 \mathrm{e} 02$ & $-7.30 \mathrm{e}-05$ & $2.98 \mathrm{e}-07$ & $-7.70 \mathrm{e}-10$ & $1.31 \mathrm{e}-12$ & $-1.49 \mathrm{e}-15$ & $1.12 \mathrm{e}-18$ & $-5.34 \mathrm{e}-22$ & $1.44 \mathrm{e}-25$ & $-1.70 \mathrm{e}-29$ \\
\hline$\left(\alpha / \mathrm{f}^{2}\right)_{\mathrm{Akh} . \mathrm{s} 1<111>}$ & -2.35 & $4.86 \mathrm{e}-02$ & $-3.28 \mathrm{e}-04$ & $1.29 \mathrm{e}-06$ & $-3.26 \mathrm{e}-09$ & $5.47 \mathrm{e}-12$ & $-6.15 e-15$ & $4.57 e-18$ & $-2.15 e-21$ & $5.78 \mathrm{e}-25$ & $-6.76 \mathrm{e}-29$ \\
\hline$\left(\alpha / \mathrm{f}^{2}\right)_{\mathrm{Akh} . \mathrm{s} 2<111>}$ & -3.69 & 0.076605 & $-5.19 \mathrm{e}-04$ & $2.06 \mathrm{e}-06$ & $-5.21 \mathrm{e}-09$ & $8.77 e-12$ & $-9.90 e-15$ & $7.38 \mathrm{e}-18$ & $-3.48 \mathrm{e}-21$ & $9.40 \mathrm{e}-25$ & $-1.10 \mathrm{e}-28$ \\
\hline
\end{tabular}


The temperature variation of non-linearity constant is presented in Figure 2 along $\langle 100\rangle$ direction. The value of $\mathrm{D}_{\mathrm{l}} / \mathrm{D}_{\mathrm{s}}$ along $<100>$ direction is about 5.34 which is expected for this type of crystals. We can see that ratio of non-linearity constants $D_{/} / D_{s}$ increases with temperature. The $\mathrm{D}$ is a measure of acoustic energy converted to thermal energy under the relaxation process, thus the increase in $\mathrm{D}_{1} / \mathrm{D}_{\mathrm{s}}$ with temperature shows that longitudinal loss increases with temperature and vice versa along $<100>$ direction. Along other two directions also the values of $D_{1}$ and $D_{s}$ decreases with temperature in a manner which shows that longitudinal loss increases with temperature.

Studies of the ultrasonic absorption in crystals and its dependence on the direction of propagation and wave mode (polarization of the elastic displacement vector) are important from the standpoint of the further development of the theory of sound wave-lattice interactions. The thermal ultrasonic attenuation along different directions is presented in Figure 3. We can see from the figure that thermal attenuation increases with temperature along all these directions and of the order of $10^{-18} \mathrm{Nps}^{2} / \mathrm{m}$ which is expected for the type of crystal under inspection. At any particular temperature the thermal attenuation along $<100>$ direction is more and is less along all other directions, hence $<100\rangle$ is more suited for the study of thermal ultrasonic attenuation. Figures 4-6 represent the ultrasonic attenuation due to phonon-phonon interaction along $<100>,<110>$ and $<111>$ crystallographic directions respectively. Along all these directions the ultrasonic attenuation increases with increase in temperature, the situation is little different for shear wave along $<110>$ direction $($ pol. $<110>$ ) for which the ultrasonic attenuation decreases with temperature. Among all these three directions the attenuation along $<111>$ direction changes sharply with temperature so the study along $<111>$ crystallographic direction is more reliable for the characterization. From the attenuation values along different directions it is evident that the ultrasonic attenuation is different along different directions i.e. varies with the orientation of the crystal.

\section{Conclusions}

From the above results one can reach to the conclusion that the thermal relaxation time, ultrasonic wave velocities and attenuation are the properties which depends on the crystallographic directions or one can say that these properties are orientation dependent which strongly supports the results given by other investigators $[14,15]$ for the same type of crystals that all these properties are strongly dependent on direction of polarization. The study of ultrasonic attenuation for longitudinal wave along $<100>$ is more important in characterization of the crystal since its magnitude is more along this direction. The rapidly increase in ultrasonic attenuation at higher temperature is interpreted mainly due to increase of density of dislocations. The results obtained in this study can be used for further investigations $[16,17]$ and industrial research and development purposes.

\section{References}

[1] R. P. Singh and R. K. Singh, "Theoretical Study of Temperature Dependent Lattice Anharmonicity in $\mathrm{TlCl}$ and T1Br," Current Applied Physics, Vol. 10, No. 4, 2010, pp. 1053-1058. doi:10.1016/j.cap.2009.12.040

[2] J. D. Pandey, A. K. Singh and R. Dey, "Effect of Isotopy on Thermoacoustical Properties," Journal of Pure and Applied Ultrasonics, Vol. 26, 2004, pp. 100-104.

[3] A. K. Upadhyay and B. S. Sharma, "Elastic Properties of Intermetallics Compounds under High Pressure and High Temperature," Indian Journal of Pure and Applied Physics, Vol. 47, 2009, pp. 362-368.

[4] R. K. Singh, "Ultrasonic Attenuation in Alkaline Earth Metals," Journal of Pure and Applied Ultrasonics, Vol. 28, 2006, pp. 59-65.

[5] S. K. Srivastava, Kailash and K. M. Raju, "Ultrasonic Study of Highly Conducting Metals," Indian Journal of Physics, Vol. 81, 2007, pp. 351-361.

[6] Kailash, K. M. Raju and S. K. Srivastava, "Acoustical Investigation of Magnesium Oxide," Indian Journal of Physics, Vol. 44, 2006, pp. 230-234.

[7] T. Yanagisaa, T. Goto and Y. Nemoto, "Ultrasonic Investtigation of Quadrupole Ordering in $\mathrm{HoB}_{2} \mathrm{C}_{2}$," Physical Review B, Vol. 67, 2003, pp. 115129-115136. doi:10.1103/PhysRevB.67.115129

[8] S. K. Kor, R. R. Yadav and Kailash, "Ultrasonic Attenuation in Dielectric Crystals," Journal of the Physical Society of Japan, Vol. 55, 1986, pp. 207-212. doi:10.1143/JPSJ.55.207

[9] A. Akhiezer, "On the Absorption of Sound in Solids," Journal of Physics-USSR, Vol. 1, 1939, p. 277.

[10] M. Born and J. M. Mayer, "Zur Gittertheorie der Lonenkristalle," Zur Physics (Germany), Vol. 75, 1932, pp. 1-18.

[11] K. Brugger, "Thermodynamic Definition of Higher Order Elastic Coefficients," Physical Review, Vol. 133, 1964, pp. A1611-A1612. doi:10.1103/PhysRev.133.A1611

[12] W. P. Mason, "Piezoelectric Crystals and Their Applications to Ultrasonics," D. Van Nostrand Co, Inc., Princeton, 1950.

[13] W. P. Mason, "Effect of Impurities and Phonon-Processes on the Ultrasonic Attenuation in Germanium, Crystal Quartz and Silicon," Physical Acoustics, Vol. 3B, Academic Press, New York, 1965, p. 237.

[14] K. M. Raju, Kailash and S. K. Srivastava, "Orientation Dependence of Ultrasonic Attenuation," Physics Proce- 
dia, International Congress on Ultrasonics, Santiago de Chile, Vol. 3, 2010, pp. 927-933.

[15] Y. Hiki and J. Tamura, "Ultrasonic Attenuation in Ice Crystals near the Melting Temperature," Journal de Physique, Vol. C5, 1981, p. 547.

[16] J. A. Scales and A. E. Malcolm, "Laser Characterization of Ultrasonic Wave Propagation in Random Media," Physical Review E, Vol. 67, 2003, pp. 046618(1) 046618(7).

[17] T. C. Upadhyay, "Temperature Dependence of Microwave Loss in ADP-type Crystals," Indian Journal of Pure and Applied Physics, Vol. 47, 2009, pp. 66-72. 